\title{
X-RAY EMISSION FROM THE SOLAR WIND
}

\author{
E. BOLDT \\ NASA, Goddard Space Flight Center, Greenbelt, Md., U.S.A. \\ and \\ A. KLIMAS* and G. SANDR I* \\ Aeronautical Research Associates of Princeton, Inc., Princeton, N.J., U.S.A.
}

\section{Introduction}

Recent developments have made it possible to detect cosmic X-rays with energies as low as several hundred $\mathrm{eV}$. Several measurements of the diffuse X-radiation in this range have been reported (Baxter et al., 1969; Bowyer et al., 1968; Henry et al., 1968). In this note we investigate the possibility that these observers have detected X-radiation emitted by the solar wind. We conclude that they probably have not. However, we also find that bremsstrahlung may be detectable from a region of the sky near the sun. If this measurement is possible, it would represent an important method for determining some characteristics of the solar wind away from the ecliptic plane of the solar system. Even though the mean free path for electron collisions is large compared with the astronomical unit, the collision frequency, electron density, and energy released per encounter are sufficient to yield detectable soft X-radiation for lines of sight close to the sun. We have estimated the expected X-ray intensity in the vicinity of the earth on the basis of two models of the solar wind flow pattern; in the first, the flow is radial in all directions away from the sun, and in the second, the flow is confined to a disc of uniform thickness near the ecliptic. In both cases, we neglect temperature gradients for the electrons and compute the total flux received from interplanetary plasma along the line of sight. Most of the received intensity comes from the segment of the line of sight which is nearest the sun. The results are insensitive with respect to the position of the boundary of the solar cavity. Accordingly, we neglect the boundary and consider an infinitely large solar cavity.

The X-radiation is assumed to arise from bremsstrahlung in an optically thin, fully ionized hydrogen gas. In the solar wind, bremsstrahlung contributions of higher mass elements (helium, in particular) should represent only a small correction. We use a standard bremsstrahlung emission formula, as presented in Tucker and Gould (1966) or Greene (1959); thus we assume that the distribution of electrons is approximately Maxwellian. The electron distribution function in interplanetary space is not well known. From the measured ion distribution, we might expect anisotropies which are characterized by different temperatures parallel and perpendicular to the interplanetary magnetic field. The anisotropy in the ion temperature is not very large, however, and

* This work was supported by National Aeronautics and Space Administration, Goddard Space Flight Center, Greenbelt, Md. under Contract No. NAS 5-11104. 
for our purpose of estimating the magnitude of the expected radiation, a more detailed analysis is not justified.

We have estimated the recombination radiation from the solar wind on the basis of the ionization equilibrium calculation done by Tucker and Gould (1966) and a universal abundance model. Recombination radiation can be, at best, comparable in intensity to the bremsstrahlung. In the following, we consider the bremsstrahlung contribution only.

\section{The Bremsstrahlung Intensity}

The differential bremsstrahlung emission rate for a volume element, $\mathrm{d} V$, of fully ionized hydrogen with density, $n$, as a function of frequency, $\omega$, and temperature, $T$, is

$$
\frac{\mathrm{d} E}{\mathrm{~d} t \mathrm{~d} V \mathrm{~d} \omega}=n^{2} \frac{2^{4}}{3^{3 / 2}} \frac{e^{6}}{m^{2} c^{3}}\left(\frac{2 \pi m}{k T}\right)^{1 / 2} \bar{g}(\omega, T) e^{-\hbar \omega / k T}
$$

where $\bar{g}(\omega, T)$ is the average Gaunt factor (Greene, 1959). For the degree of accuracy desired here, we may set $\bar{g}(\omega, T)=1$. The power received at a detector of surface area, $\mathrm{d} A$, with beam width solid angle, $\mathrm{d} \Omega$, from a distributed source in which the detector is embedded is

$$
\frac{\mathrm{d} E_{\mathrm{R}}}{\mathrm{d} t \mathrm{~d} \omega \mathrm{d} A \mathrm{~d} \Omega}=\frac{1}{4 \pi} \int_{0}^{\infty} \mathrm{d} l\left(\frac{\mathrm{d} E}{\mathrm{~d} t \mathrm{~d} V \mathrm{~d} \omega}\right)
$$

where the integration is carried out along the line of sight. Assuming a uniform temperature for the electrons, we obtain

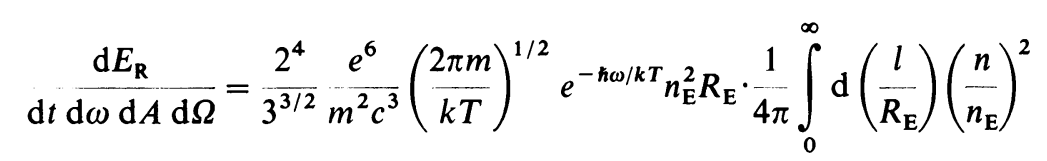

where $n_{\mathrm{E}}$ is the electron density at the earth and $R_{\mathrm{E}}$ is $1 \mathrm{AU}$. Equation (3) can be written numerically as

$$
\begin{aligned}
& \frac{\mathrm{d} E_{\mathrm{R}}}{\mathrm{d} t \mathrm{~d}(\hbar \omega) \mathrm{d} A \mathrm{~d} \Omega}=1.24 \times 10^{-4} n_{\mathrm{E}}^{2}\left(\frac{m c^{2}}{k T}\right)^{1 / 2} e^{-\hbar \omega / k T} \\
& \cdot F(\hat{l}) \quad\left(\mathrm{keV} / \mathrm{keV} \mathrm{sec} \mathrm{cm} \mathrm{cm}^{2} \text { ster }\right)
\end{aligned}
$$

where

$$
F(\hat{l})=\frac{4}{\pi} \int_{0}^{\infty} \mathrm{d}\left(\frac{l}{R_{\mathrm{E}}}\right)\left(\frac{n}{n_{\mathrm{E}}}\right)^{2}
$$

can only be a function of the direction of the line of sight, symbolized by $\hat{l}$. In Table I we list the value given by Equation (4), if $F(\hat{l})=1$, for some pertinent values of $\omega$, $T_{6}=10^{-6} T(\mathrm{~K})$ and $n_{\mathrm{E}}$. In the remainder of this paper we calculate $F(\hat{l})$ for two models of the solar wind flow. The power received from a given direction at a particular temperature, density, and frequency can be obtained by multiplying the appropriate value of $F(\hat{l})$ from Figure 1 by the appropriate number from Table I. 
TABLE I

\begin{tabular}{|c|c|c|c|c|}
\hline$\hbar \omega(\mathrm{eV})$ & $T_{6}$ & $n \mathbf{E}$ & $\mathrm{d} E_{\mathrm{R}} / \mathrm{d} t \mathrm{~d}(\hbar \omega) \mathrm{d} A \mathrm{~d} \Omega$ & $\mathrm{keV} /\left(\mathrm{keV} \mathrm{sec} \mathrm{cm}^{2}\right.$ ster $)$ \\
\hline \multirow[t]{3}{*}{100} & 0.5 & 1 & $1.34 \times 10^{-3}$ & \\
\hline & & 5 & $3.36 \times 10^{-2}$ & \\
\hline & & 80 & 8.60 & \\
\hline \multirow[t]{3}{*}{100} & 1 & 1 & $3.01 \times 10^{-3}$ & \\
\hline & & 5 & $7.53 \times 10^{-2}$ & \\
\hline & & 80 & 19.3 & \\
\hline \multirow[t]{3}{*}{100} & 2 & 1 & $3.80 \times 10^{-3}$ & \\
\hline & & 5 & $9.50 \times 10^{-2}$ & \\
\hline & & 80 & 24.3 & \\
\hline \multirow[t]{3}{*}{250} & 0.5 & 1 & $4.09 \times 10^{-5}$ & \\
\hline & & 5 & $1.02 \times 10^{-3}$ & \\
\hline & & 80 & $2.62 \times 10^{-1}$ & \\
\hline \multirow[t]{3}{*}{250} & 1 & 1 & $5.28 \times 10^{-4}$ & \\
\hline & & 5 & $1.32 \times 10^{-2}$ & \\
\hline & & 80 & 3.38 & \\
\hline \multirow[t]{3}{*}{250} & 2 & 1 & $1.60 \times 10^{-3}$ & \\
\hline & & 5 & $3.99 \times 10^{-2}$ & \\
\hline & & 80 & 10.2 & \\
\hline
\end{tabular}

\section{The Models}

In both models of the solar wind flow we neglect the small variation of solar wind speed near the sonic point (Parker, 1963). Thus, the radial dependence of the solar wind density can be obtained from kinematic considerations. If the flow is spherical, $n \propto R^{-2}$, and if the flow is cylindrical, $n \propto R^{-1}$. The effect of neglecting wind speed variation near the sun is to underestimate the expected $X$-ray intensity from a small region of the sky very near the sun.

In the cylindrical flow model we calculate $F(\hat{l})$ for directions in the ecliptic. Thus, the line of sight remains in the gas disc. For directions out of the ecliptic, we would expect the X-ray intensity to decrease more rapidly with increasing angular distance from the sun, but the details of this rapid decrease would depend on the disc structure. In any case, we would expect the actual data to clearly differentiate the cylindrical from the spherical flow.

We set

$$
n=n_{\mathrm{E}}\left(R_{\mathrm{E}} / R\right)^{\alpha}
$$

where $\alpha=1$ is the cylindrical flow and $\alpha=2$ is the spherical flow. Then

$$
F_{\alpha}(\hat{l})=\frac{4}{\pi} \int_{0}^{\infty} \mathrm{d}\left(\frac{l}{R_{\mathrm{E}}}\right)\left(\frac{R_{\mathrm{E}}}{R}\right)^{\alpha} .
$$

If $\theta$ is the angle between the line of sight in the ecliptic and the earth-sun line, then

$$
\frac{R}{R_{\mathrm{E}}}=1+\left(\frac{l}{R_{\mathrm{E}}}\right)^{2}-2 \frac{l}{R_{\mathrm{E}}} \cos \theta .
$$


We find

and

$$
F_{1}(\theta)=\frac{4}{\pi}\left(\frac{\pi-\theta}{\sin \theta}\right)
$$

$$
F_{2}(\theta)=\frac{2}{\pi \sin ^{2} \theta}\left[\cos \theta+\frac{\pi-\theta}{\sin \theta}\right] .
$$

For $\theta \ll 1$,

$$
F_{1}(\theta) \propto 1 / \theta \text { and } F_{2}(\theta) \propto 1 / \theta^{3}
$$

and, for $\theta=\pi / 2$,

$$
F_{1}(\theta)=2 \text { and } F_{2}(\theta)=1 .
$$

$F_{1}(\theta)$ and $F_{2}(\theta)$ are plotted in Figure 1.

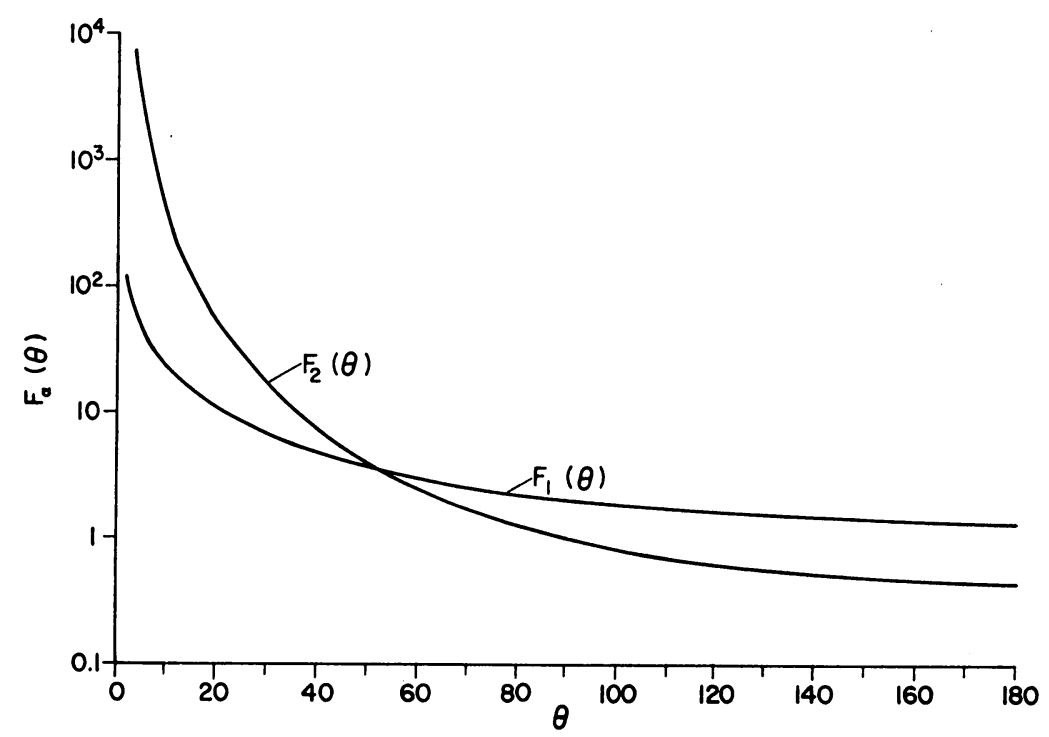

Fig. 1. The functions $F_{1}(\theta)$ and $F_{2}(\theta)$.

\section{Discussion}

We see that, except for small $\theta, F_{1}(\theta)$ and $F_{2}(\theta)$ are slowly varying functions which remain close to the value, one. A scan of Table I for $\hbar \omega=250 \mathrm{eV}$ clearly reveals that the X-ray intensity expected from the solar wind over most of the sky is small compared to the $50 \mathrm{keV} / \mathrm{keV} \mathrm{cm} \mathrm{cm}^{2} \mathrm{sec}$ ster which have been observed from the diffuse $\mathrm{X}$-radiation (Kraushaar, this volume). On the other hand, the steep rise of $F_{1}(\theta)$ and $F_{2}(\theta)$ for small $\theta$ makes it likely that bremsstrahlung from a small region of the sky near the sun could be detected. To reduce the extragalactic background, observations should be made when the sun is at low galactic latitudes in the sky, 
where absorption in the interstellar gas will strongly attenuate the diffuse radiation. The necessary sensitivity to observe a predetermined region near the sun can be obtained from Table I and Figure 1. For example, consider the following quiet sun conditions:

$$
\begin{aligned}
& n_{\mathrm{E}}=5 \mathrm{~cm}^{-3} \\
& T_{6}=1 .
\end{aligned}
$$

For $\hbar \omega=250 \mathrm{eV}$, at approximately $\theta=5^{\circ}$, we obtain the intensity, $50 \mathrm{keV} / \mathrm{keV} \mathrm{cm}{ }^{2}$ sec ster. This intensity is comparable to the diffuse background intensity quoted above ; however, the strong interstellar absorption at low galactic latitudes should prevent confusion of the two sources. During periods of high solar activity, when larger $n_{\mathrm{E}}$ are observed, the X-radiation from the solar wind would be more intense, and observable over a larger portion of the sky.

\section{References}

Baxter, A. J., Wilson, B. G., and Green, D. W.: 1969, Astrophys. J. (Letters) 155, L145.

Bowyer, C. S., Field, G. B., and Mark, J. E.: 1968, Nature 217, 32.

Greene, J.: 1959, Astrophys. J. 130, 693.

Henry, R. C., Fritz, G., Meekins, J. F., Friedman, H., and Byram, E. T.: 1968, Astrophys. J. (Letters) 153, L11.

Parker, E. N.: 1963, Interplanetary Dynamical Processes, Interscience, New York and London, pp. 51-72.

Tucker, W. H. and Gould, R. J.: 1966, Astrophys. J. 144, 244. 